\title{
EDUCACIÓN GEOGRÁFICA INTERCULTURAL EN LA FORMACIÓN Y EL DESARROLLO PROFESIONAL DEL PROFESORADO: UNA REVISIÓN DE LA LITERATURA
}

\section{INTERCULTURAL GEOGRAPHIC EDUCATION IN THE TRAINING AND PROFESSIONAL DEVELOPMENT OF TEACHERS: A LITERATURE REVIEW}

Daniel Llancavil Llancavil*

RESUMEN: Este artículo revisa la literatura internacional sobre educación geográfica intercultural en la formación y desarrollo profesional del profesorado de Geografía. Utilizando un enfoque de revisión por alcance, se realizó una búsqueda en diversas bases de datos desde donde se recopilaron 234 artículos, considerando las palabras claves: educación geográfica, didáctica de la geográfica, educación geográfica intercultural, interculturalidad y educación intercultural. Para sistematizar las publicaciones encontradas se utilizó el gestor bibliográfico Mendeley. Luego de aplicar los criterios de inclusión y exclusión se seleccionaron 58 artículos, realizándose un análisis de contenido de cada publicación a partir del cual se establecieron dos dimensiones para la presentación de los resultados: educación geográfica e interculturalidad. Para la primera de éstas se crearon las categorías de pedagogía, disciplina y didáctica, mientras que para la segunda las de competencia intercultural, educación intercultural y prácticas de enseñanza interculturales. Los hallazgos de la revisión por alcance realizada permiten evidenciar que existen escasas investigaciones en torno a educación geográfica intercultural, tanto a nivel nacional como internacional. Además, existe un importante número de estudios internacionales referidos a interculturalidad y educación intercultural $y$, en menor cantidad, sobre didáctica y enseñanza de la geografía. Se concluye que existe un importante campo de investigación aún por explorar por parte de la didáctica de la geografía. La ampliación de estudios en este ámbito contribuiría a la formación inicial docente y a la formación continua de los profesores en ejercicio, favoreciendo el desarrollo de las competencias necesarias para establecer salas de clases interculturales e inclusivas.

Palabras clave: Educación geográfica, interculturalidad, didáctica de la geografía, formación docente. 


\begin{abstract}
This article reviews the international literature on intercultural geographical education in the training and professional development of geography teachers. Using a scoping review approach, a search was conducted in several databases from which 234 articles were collected considering the keywords: 'educación geográfica', 'didáctica de la geográfica', 'educación geográfica intercultural', 'interculturalidad' and 'educación intercultural' ['geographical education, 'didactics of geography', 'intercultural geographical education', 'interculturality' and 'intercultural education']. The bibliographic manager Mendeley was used to systematize the publications found. After applying the inclusion and exclusion criteria, 58 articles were selected and a content analysis of each publication was carried out, from which two dimensions were established for the presentation of the results: geographic education and interculturality. For the former, the categories of pedagogy, discipline and didactics were established, while for the latter, intercultural competence, intercultural education and intercultural teaching practices. The findings of the review by scope carried out show that there is little research on intercultural geographic education both nationally and internationally. In addition, there is an important number of international studies on interculturality and intercultural education and, to a lesser extent, on geography didactics and teaching. It is concluded that there is an important field of research yet to be explored by geography didactics. The expansion of studies in this field would contribute to the initial teacher training and to the continuing education of practicing teachers, favoring the development of the necessary competencies to establish intercultural and inclusive classrooms.
\end{abstract}

Key words: Geographic education, interculturality, geography didactics, teacher training.

\title{
INTRODUCCIÓN
}

Cada día las aulas escolares de los diferentes países del mundo reúnen a estudiantes de diversas nacionalidades y culturas, evidenciándose una significativa multiculturalidad en estos espacios educativos. Este escenario plantea el desafío de generar aulas interculturales e inclusivas a través de la mediación de los profesores. De ahí la importancia de enfocar la mirada en la formación inicial de los futuros profesores de modo de prepararlos para enseñar en un contexto de diversidad. La cultura y la interculturalidad son parte fundamental de la geografía y constituyen fenómenos que ésta estudia y cuya relación justifica la importancia de pensar en una educación intercultural desde la educación geográfica a partir de los primeros años de la educación escolar. Lo anterior, constituye un desafío ineludible para la didáctica de la geografía en torno a desarrollar orientaciones didácticas que propicien un dialogo y una convivencia intercultural en las aulas, proyectando esta coexistencia hacia otras dimensiones del espacio geográfico. Es fundamental que los estudiantes, desde sus primeros años de escolaridad, puedan explicar las relaciones entre los componentes del espacio geográfico para construir una visión intercultural de éste en diferentes escalas, movilizar conceptos, habilidades y actitudes en situaciones de la vida cotidiana y participar de manera informada, reflexiva y crítica en el espacio donde se habita. Por lo anterior, el gran desafío es generar un 
cuerpo de conocimientos que permita desarrollar una educación geográfica intercultural en las salas de clases.

La educación geográfica en Chile no goza de un protagonismo curricular, concentrándose sólo en los primeros niveles de la educación primaria. En los niveles superiores, los temas geográficos, anclados en la asignatura de Historia, Geografía y Ciencias Sociales, van perdiendo presencia en favor de las otras disciplinas. Lo anterior, genera un distanciamiento de los estudiantes con respecto a la geografía y una escasa comprensión y valoración de ésta para explicar las dinámicas sociales desde una dimensión espacial. A pesar de este desalentador escenario es posible encontrar, en los últimos quince años en Chile, publicaciones referidas a la enseñanza de la geografía. Entre éstas destacan estudios orientados hacia la sustentabilidad de los espacios geográficos y el rol de la didáctica (Araya, 2010); las estrategias de enseñanza y el desarrollo del pensamiento geográfico (Araya y Álvarez, $2019)$ y a las innovaciones didácticas en la enseñanza universitaria de la geografía (Arenas y Salinas, 2013). Además, existen publicaciones sobre el trabajo de campo a partir de la indagación (Lozano, 2010; Llancavil, 2018l, la relación entre educación geográfica y formación ciudadana (Arenas et al., 2018), el saber espacial informal en los espacios escolares (Garrido, 2005) y la presencia histórica de la geografía en el currículo escolar chileno (Miranda, 2012). A su vez, la Sociedad Chilena de Ciencias Geográficas, a través de sus anales y congresos, ha dado cabida a trabajos vinculados a la educación geográfica y ambiental.

Por otra parte, los estudios sobre educación intercultural, principalmente en contexto mapuche, son abundantes en nuestro país.
Sin embargo, las investigaciones sobre educación geográfica intercultural son escasas y generales. Entre éstas se encuentran los trabajos de Williamson y Pérez (2017), sobre educación ambiental intercultural; de Quintriqueo et al., (2015), sobre la enseñanza de la Historia, Geografía y Ciencias Sociales, desde una perspectiva intercultural y de Becerra y Llanquinao (2017), sobre las relaciones mapuche entre persona, tiempo y espacio. Esta exigua producción en torno a educación geográfica intercultural deja abierto un enorme campo de investigación por cubrir y genera la necesidad de conocer cuál es el estado del arte con respecto a esta temática en otros países del mundo.

A partir de lo anterior, este estudio tiene como objetivo indagar sobre las publicaciones realizadas entre 2016 y 2020 en diferentes países del mundo sobre educación geográfica intercultural. De este modo, busca dar respuesta a la siguiente pregunta: ¿Cuáles son las investigaciones sobre Educación Geográfica intercultural publicadas durante los últimos cinco años? A partir de ella se desprenden las siguientes interrogantes ¿Cuáles son las temáticas más consideradas en estas publicaciones? ¿En qué países se concentra el mayor volumen de producción? ¿Cuáles son las preguntas que deberán ser investigadas en los próximos años? Para lo anterior, se realizó una revisión por alcance para determinar la cobertura de la literatura existe sobre el tema en estudio. De acuerdo con Müun et al. (2018), este enfoque es una herramienta útil para mapear e identificar la información disponible. De este modo, se buscó ampliar las evidencias recolectadas a la fecha y así conformar un marco referencial más completo y actualizado. 


\section{MÉTODOS}

Este trabajo utilizó un enfoque de revisión disponibles, así como una descripción por alcance. Las revisiones por alcance general (amplia o detallada) de su son una herramienta ideal para determinar enfoque. En este sentido, el propósito la cobertura de un cuerpo de literatura general de realizar revisiones de alcance sobre un tema dado y entregar una visión es identificar y mapear la evidencia clara del volumen de literatura y estudios disponible (Müun et al., 2018).

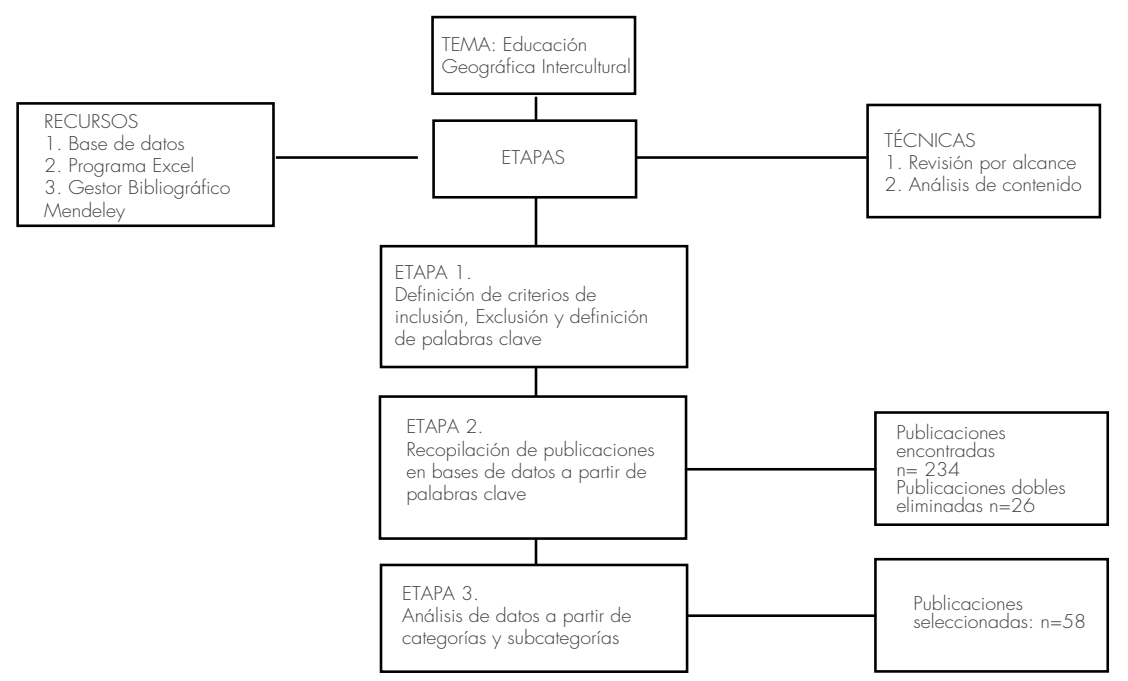

Figura 1. Diagrama del proceso desarrollado durante la revisión por alcance. (Fuente: elaboración propia)

\section{CRITERIOS DE INCLUSIÓN Y EXCLUSIÓN}

El primer paso en esta revisión por alcance antagónica de un concepto, pero de esta fue establecer los criterios de inclusión y manera se puede asignar un criterio de exclusión (ver Tabla 1). Es importante aceptación o rechazo a cada artículo del definir tanto unos como otros, aunque conjunto de datos que se está manejando muchas veces sean la expresión (García, 2018).

\section{Criterios de inclusión}

A. El artículo debe hablar de Educación Geográfica Intercultural, Educación Geográfica, Didáctica de la Geografía, Interculturalidad o Educación Intercultural.

B. El artículo debe estar situado en la formación inicial docente o enseñanza en aula.

C. El artículo debe hacer sido publicado hasta 5 años atrás (2016-2020).

\section{Criterios de exclusión}

A. Los artículos externos al ámbito de la formación inicial docente y enseñanza en aula.

B. Publicaciones anteriores al año 2016.

C. Libros, capítulos de libro, tesis, actas de congresos. 


\section{ESTRATEGIA PARA LA BÚSQUEDA DE LITERATURA}

Primero se realizó una búsqueda geografía" "Educación Intercultural", "Interelectrónica manual de varias bases de culturalidad", "Educación geográfica interdatos: Dialnet, ERIC, Intech Open, JSTOR, cultural". Se identificaron 234 estudios (ver ProQuest, RRL, REDIB, SciELO, ScopuS, Tabla 2) y se eliminaron 26 artículos Taylor \& Francis,

WOS, dobles. En total, 58 estudios cumplieron los utilizando las siguientes palabras clave: criterios de inclusión y fueron utilizados en "Educación geográfica", "Didáctica de la los análisis (ver Tabla 3).

\begin{tabular}{lclc}
\hline $\begin{array}{c}\text { Búsqueda inicial (base } \\
\text { de datos) }\end{array}$ & Número de estudios & $\begin{array}{c}\text { Búsqueda } \\
\text { complementaria } \\
\text { Referencia de estudios }\end{array}$ & Número de estudios \\
\hline Dialnet & 16 & $\begin{array}{l}\text { Estudios publicados } \\
\text { antes de enero de }\end{array}$ & 98 \\
ERIC & 2016. & Estudios en Inglés u otro & 46 \\
Intech Open & 1 & idioma diferente al & \\
JSTOR & 6 & & \\
ProQuest & 1 & & \\
RRL & 1 & & \\
REDIB & 14 & & \\
SciELO & 11 & & \\
Scopus & 2 & & \\
Taylor \& Francis & 28 & & \\
WOS & 6 & & \\
WOL & 2 & & \\
\hline
\end{tabular}

Tabla 2. Resultados de la búsqueda en diferentes bases de datos.

(Fuente: Elaboración propia)

\begin{tabular}{|c|c|c|}
\hline Base de datos & $\begin{array}{l}\text { Número de } \\
\text { estudios }\end{array}$ & País de publicación \\
\hline Taylor \& Francis & 24 & $\begin{array}{l}\text { Reino Unido (20), Turquía (1), España (1), Chipre (1), } \\
\text { Estados Unidos (1). }\end{array}$ \\
\hline Scielo & 14 & $\begin{array}{l}\text { Chile (5), México (5), Brasil (1), Costa Rica (1), Colombia } \\
\text { (1), Perú (1). }\end{array}$ \\
\hline Dialnet & 11 & España (6), Brasil (2), Colombia (2), Argentina (1). \\
\hline JSTOR & 3 & Reino Unido (2), Países Bajos (1). \\
\hline REDIB & 3 & Brasil (2), Argentina (1). \\
\hline Intech Open & 1 & Reino Unido \\
\hline ERC & 1 & Suiza \\
\hline Web Of Science & 1 & Colombia \\
\hline
\end{tabular}

Tabla 3. Estudios seleccionados según criterios de inclusión y exclusión.

(Fuente: Elaboración propia) 


\section{DESARROLLO}

La revisión de la información recopilada se realiza a partir de la presentación de dos dimensiones relacionadas con la temática central de este estudio: educación geográfica e interculturalidad. La revisión de cada artículo asociado a estas dimensiones permitió el levantamiento de categorías para cada una de ellas.

\section{DIMENSIÓN 1: EDUCACIÓN GEOGRÁFICA}

La Educación Geográfica integra el saber pedagógico y el conocimiento geográfico, preocupándose del desarrollo del pensamiento espacial y la formación de una racionalidad geográfica que indague sobre las problemáticas sociales y ambientales que afectan al ser humano en su vinculación con la naturaleza (Araya y De Souza, 2018). Se ha situado en dos perspectivas temáticas: la primera de ellas, educación geográfica para la sustentabilidad, llamada a responder a una demanda con profundas connotaciones sociales, económicas y ambientales; la segunda de ellas, educación geográfica para la formación ciudadana, entrega a los estudiantes la posibilidad para desarrollar actividades que promuevan un cambio de actitud y una valoración hacia el entorno local, transformándose en ciudadanos responsables que se vinculan activa y favorablemente con el espacio geográfico (Araya, 2010).

Constituye una subdisciplina de la didáctica general que se nutre del saber pedagógico y del conocimiento geográfico. Inicialmente su foco estaba centrado en las estrategias para mejorar la enseñanza de los conocimientos geográficos. Hoy, debido al avance de la psicología educacional y otras disciplinas preocupadas del desarrollo cognitivo, el foco se ha trasladado hacia el mejoramiento de los procesos de aprendizaje de los estudiantes.

Para esta dimensión se seleccionaron dieciséis artículos cuya revisión permitió levantar las categorías que se presentan en la Tabla 4.

\begin{tabular}{|c|c|c|}
\hline Dimensión & Categoría & Estudios \\
\hline & Pedagogía & $\begin{array}{l}\text { Applis (2016); Karolčík, Čipková \& Mázorová (2016); } \\
\text { Salinas \& Brooks (2018); Scholten, Höttecke \& Sprenger } \\
\text { (2019); Brysch (2020); Golightly (2021); Puttick \& } \\
\text { Warren-Lee (2021); }\end{array}$ \\
\hline & Disciplina & $\begin{array}{l}\text { Alomar, Gómez \& Arias (2017); Béneker \& Palings } \\
\text { (2017); Brooks (2017); Martinez (2017); Nguyen } \\
\text { (2018); Fögele, Luber \& Rainer (2019). }\end{array}$ \\
\hline Geográfica & Didáctica & $\begin{array}{l}\text { Harte (2017); Araya \& De Souza (20 1 8); Svobodová, } \\
\text { Mísařová, Durna \& Hofmann (2020). }\end{array}$ \\
\hline
\end{tabular}

Tabla 4. Resultados de la búsqueda de datos sobre la dimensión educación geográfica. (Fuente: Elaboración propia) 


\section{CATEGORÍA: PEDAGOGÍA}

Diversas investigaciones analizan las prácticas docentes en la enseñanza de la geografía (Applis, 2016; Salinas y Brooks, 20181. Estas destacan que el desarrollo teórico de la didáctica de la disciplina provee a los profesores referentes para atender las problemáticas del contexto educativo tomando como punto de apoyo su "experticia" en geografía. Asimismo, éste es relevante para estrechar lazos entre la disciplina y las experiencias de aprendizaje de los estudiantes, situando la capacidad de agencia del docente en su rol como especialista articulador de conocimientos y los espacios que les dan significado (Salinas y Brooks, 2018). En Europa existen varias investigaciones en torno al tema. Una de éstas indagó sobre la incorporación de valores reflexivos en las clases de geografía, destacando la importancia de considerar esta dimensión en la formación docente y en las prácticas de los profesores alemanes en ejercicio (Applis, 2016).

Una investigación en Alemania analizó la capacidad de los profesores de geografía para reconocer diversos estímulos durante la instrucción (Scholten et al., 2019). Los autores sostienen que para enseñar de manera receptiva, los docentes deben poder identificar y abordar los incidentes del aula que son críticos para el aprendizaje de los estudiantes. Por lo anterior, resulta valioso indagar sobre la percepción e interpretación de los docentes de tales incidentes, así como la toma de decisiones asociadas. Los hallazgos indican que la mayoría de los profesores percibieron los incidentes y expresaron diversas interpretaciones sobre estos. Este estudio también representa un avance metodológico, pues la investigación basada en videos sigue siendo poco común en educación geográfica. En Inglaterra, se investigó sobre la retroalimentación de las lecciones realizada por mentores a profesores principiantes de geografía (Puttick y Warren-Lee, 20211. A partir de aquello, los autores hacen sugerencias para mejorar la atención en la retroalimentación de las lecciones, centrando la atención en el progreso, la explicación y terminología utilizada. En Eslovaquia, destaca una investigación que indaga sobre las opiniones y actitudes de los profesores de Geografía sobre programas de perfeccionamiento referidos a la innovación y modernización de los procedimientos, métodos y formas de enseñanza de la geografía con la ayuda de tecnologías digitales (Karolčík et al., $2016)$. En ella, los docentes manifestaron una positiva valoración a este tipo de programas según su nivel, contenido y beneficio general de los módulos educativos individuales.

Por otra parte, también fue posible encontrar un reciente estudio sobre las percepciones de profesores sudafricanos en formación con respecto a la colaboración y las interacciones productivas entre futuros docentes de geografía (Golightly, 2021). Este incorporó la autoevaluación y la evaluación de pares en una actividad de aprendizaje basado en problemas. Los resultados indicaron que el esfuerzo, la actitud y la colaboración recibieron las puntuaciones más altas en la autoevaluación y en la evaluación de los profesores de geografía en formación. Asimismo, estos otorgaron puntuaciones más altas por su contribución a sí mismos, en comparación con la evaluación de pares y no se produjeron diferencias reales de género. Finalmente, un estudio 
norteamericano estableció que algunos profesores estadounidenses de geografía, ante instancias de capacitación, todavía prefieren los talleres presenciales. Sin embargo, muchos de ellos están dispuestos a aceptar y participar en el desarrollo profesional basado en la web con un enfoque híbrido, donde se combinan métodos cara a cara y en línea (Brysch, 2020).

En esta categoría se evidencian aspectos pedagógicos que los futuros profesores y los docentes en ejercicio deben tener en cuenta al momento de realizar sus clases de geografía. En este sentido, se revela la importancia de considerar las problemáticas del contexto en los cuales se enseña, como también reconocer los incidentes críticos que pueden desarrollarse al interior de la sala de clases para así poder llevar a cabo un adecuado ejercicio pedagógico. Asimismo, se demanda que los profesores tengan la capacidad de incorporar valores reflexivos en sus clases. Además, destaca la importancia de establecer comunidades de apoyo entre los docentes con el fin de estimular y propiciar la retroalimentación entre pares. Todo lo anterior son insumos relevantes de considerar en la formación inicial docente y posteriormente durante el desarrollo profesional.

\section{CATEGORÍA: DISCIPLINA}

Una investigación española presenta una reflexión acerca del interés y sentido de la enseñanza de la Geografía en la formación universitaria de los futuros maestros españoles (Martínez, 2017). Deja en evidencia que la reciente reforma a los estudios de grado han disminuido las materias de "contenidos" favoreciendo, de manera explícita, a las de índole didáctico-pedagógico. Lo anterior, señala el autor, ha ido en perjuicio de la Geografía General, del Mundo o de España, como materias propias de la disciplina en la totalidad de las titulaciones ofertadas por las universidades públicas españolas. En la misma dirección, otro estudio sostiene que es importante que los futuros profesores cuenten con el apoyo de especialistas en geografía durante su formación inicial. Lo anterior les permitirá desarrollar una fuerte identidad geográfica y una perspectiva disciplinaria sobre por qué es importante enseñar geografía (Brooks, 2017).

Por otra parte, un estudio en Alemania demostró que existen orientaciones profundamente arraigadas en los profesores con respecto a las clases de geografía que impactan en la práctica futura en el aula y el aprendizaje en la universidad. En este sentido, se reconocieron cuatro tipos diferentes de estudiantes de profesorado: "Estudio orientado al logro", "Estudio orientado a la aplicación", "Estudio orientado a la experiencia" y "Estudio orientado a la cognición". Esta tipología permite a los profesores entrar en procesos de reflexión con los futuros profesores sobre sus orientaciones (Fögele et al., 2019).

Si consideramos contenidos propios de la disciplina, encontramos una investigación holandesa que analiza las ideas de los futuros profesores holandeses con respecto a qué tipo de conocimiento geográfico deberían aprender los estudiantes de secundaria (Béneker y Palings, 2017). Experiencias similares se encuentran en Vietnam, donde uno de los estudios revisados explora las perspectivas de los profesores de geografía sobre una educación para el desarrollo sostenible (Nguyen, 2018). Asimismo, en España se 
indagó sobre la enseñanza del paisaje en el nuevo plan de estudios de los Grados de Geografía, evidenciando la necesaria actualización de la enseñanza del paisaje a nivel universitario en el contexto de la Geografía, con miras a adecuar tanto la práctica profesional como la investigación (Alomar-Garau et al., 2017).

Esta segunda categoría nos lleva a debatir sobre qué conocimientos tienen mayor protagonismo en la formación del futuro profesor de geografía, si los contenidos disciplinares o los didáctico-pedagógicos. Sin tener la necesidad de tener que zanjar esta disyuntiva en este escrito, conviene señalar que existe un consenso en torno al hecho de que el futuro profesor y el docente en ejercicio tengan un dominio disciplinar de la materia que enseñan. Para lo anterior, se requiere que la formación disciplinar, durante su paso por las carreras de pedagogía, esté en manos de especialistas disciplinarios, idealmente con una formación pedagógica y didáctica. Esto lleva aparejado también la necesidad de una permanente actualización de saberes, de manera autónoma $\mathrm{o}$ en programas de Educación Continua. Asimismo, la enseñanza de la disciplina debe ir acompañada de una permanente reflexión sobre lo que se enseña.

\section{CATEGORÍA: DIDÁCTICA}

En la República Checa, se indagó sobre los beneficios de la educación al aire libre en la enseñanza de la geografía según cómo la perciben los maestros, alumnos y padres (Svobodová et al., 2020). La investigación mostró que los docentes ven los beneficios tanto en el área cognitiva como en el aspecto afectivo de la educación y saben que los estudiantes adquieren ciertas habilidades y conocimientos. En cambio, los padres ven el mayor beneficio en el área de actitudes y relaciones interpersonales. Por último, los alumnos perciben la educación al aire libre como entretenimiento y como tiempo que pasan fuera del aula en lugar de un proceso de aprendizaje. Un estudio realizado en Brasil, buscó indagar en los procesos de construcción del pensamiento geográfico en 45 estudiantes de profesorado en Geografía de la Universidad Federal de Goías (Araya y De Souza, 2018). Entre los principales resultados, se aprecia que los trabajos didácticos contienen actividades que, según los planteamientos de Vigotsky, permiten el desarrollo de conceptos y habilidades de pensamiento superior, con énfasis en procesos de razonamiento geográfico. En Australia, se evaluó la eficacia de las experiencias de aprendizaje de tecnología geoespacial (GT) en dos cursos del plan de estudios de geografía para determinar su efectividad para desarrollar la confianza de los maestros en formación y prepararlos para incorporar GT en sus prácticas de enseñanza (Harte, 2017). Los resultados indican que la confianza y las competencias de los profesores en formación, para integrar con éxito la GT en su enseñanza, aumentaron durante la duración del estudio. Los hallazgos destacan el valor de brindar experiencias de aprendizaje de GT en los cursos del plan de estudios de geografía.

Con respecto a esta categoría es posible afirmar, a partir de los estudios revisados, que prima una importante renovación de cómo entender la didáctica de la geografía hoy en día y cómo las metodologías de enseñanza se han movilizado hacia estrategias más interactivas. Hay un interés por indagar en 
cómo desarrollar el pensamiento geográfi- relacionan. Bajo este contexto, la co en los estudiantes para que estos interculturalidad busca la confluencia de las puedan comprender su espacio geográfico culturas y una articulación de las diferencias a partir de estrategias como las excur- bajo el aprendizaje mutuo, la cooperación y siones al aire libre y el uso de herramientas el intercambio (López, 2001). Desde esta tecnológicas de referenciación geoespa- perspectiva, la educación, la escuela y el ciales.

\section{DIMENSIÓN 2: INTERCULTURALIDAD}

El concepto de interculturalidad hace referencia a "una situación, proceso o proyecto que se produce en el espacio entre distintas formas de producción cultural" (Novaro, 2006, p.2). En ella se producen encuentros entre grupos humanosdiferentes, en donde se entrecruzan Se seleccionaron treinta y seis artículos para los sistemas simbólicos y generan transforma- esta dimensión cuya revisión permitió levantar ciones culturales entre los grupos que se las categorías que se presentan en la Tabla 5.

\begin{tabular}{|c|c|c|}
\hline Dimensión & Categoría & Estudios \\
\hline \multirow{3}{*}{ Interculturalidad } & Competencia intercultural & $\begin{array}{l}\text { Brendel et al. (2016); Escarbajal } \\
\text { \& Morales (2016); Fernández et } \\
\text { al. (2016); Hernández (2016); } \\
\text { Moreno \& Atienza (2016); } \\
\text { Shestakova et al. (2016); Dervin } \\
\text { (2017); Siqueira (2017); } \\
\text { Garrote et al. (2018); Mendes \& } \\
\text { Lustosa (2018); Vea \& Leirvik } \\
\text { (2018); Senyshyn (2018); } \\
\text { Morales-Saavedra et al. (2018); } \\
\text { Loo et al. (2019); Aziz et al. } \\
\text { (2020); Garrard (2020); Nunes } \\
\text { \& Lima (2020); Rezende \& } \\
\text { Rodríguez (2020). }\end{array}$ \\
\hline & Educación intercultural & 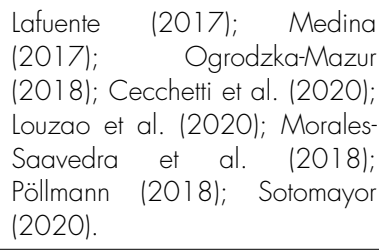 \\
\hline & $\begin{array}{l}\text { Prácticas de enseñanza } \\
\text { interculturales }\end{array}$ & $\begin{array}{l}\text { Escarbajal \& Morales }(2016) \text {; } \\
\text { Rodríguez-lzquierdo }(2016) \text {; } \\
\text { Vranješević \& Frost }(2016) \text {; } \\
\text { Bazan \& Tejerina (2018); } \\
\text { lbáñez-Salgado \& Druker-lbáñez } \\
(2018) \text { Karousiou et al. } \\
(2018) \text {; Becerra-Lubies et al. } \\
(2019) ; \text { Beltrán- Véliz et al. } \\
(2019) \text { Sacavino (2020); } \\
\text { Gómez- Parra \& Há (2021). }\end{array}$ \\
\hline
\end{tabular}

Tabla 5. Resultados de la búsqueda de datos sobre la dimensión educación intercultural (Fuente: Elaboración propia) 


\section{CATEGORÍA: COMPETENCIA INTERCULTURAL}

En esta categoría fue posible encontrar estudios asociados a la formación del profesorado en niveles de enseñanza primaria, secundaria y universitaria en diversos países y continentes. Investigaciones sobre las percepciones de los docentes de educación primaria sobre la competencia intercultural en España y Norvega demuestran diversas actitudes con respecto a ésta. Un estudio demostró que los profesores españoles poseen una gran disposición para trabajar con estudiantes de otras culturas. Asimismo, se evidencia una relación positiva entre los años experiencia laboral y el trabajo de esta competencia en el aula (Garrote ef al., 20181. Otro estudio en aquel país dio cuenta de la necesidad de trabajar de manera transversal esta competencia en la formación del profesorado en educación intercultural. Lo anterior permitiría enseñar a educar desde un punto de vista no etnocentrista para así evitar conflictos culturales y conseguir una cohesión social que permita el diálogo intercultural (Moreno y Atienza, 2016). Por su parte, una investigación desarrollada en Norvega, estableció que los procesos formativos no ponen énfasis en la competencia intercultural, incluida la conciencia cultural sin estereotipos y atributos personales como la apertura y la empatía. De ahí la necesidad de insistir en procesos de formación inicial docente orientados a la comprensión de la competencia intercultural de los profesores en el diverso entorno educativo norvego (Vea y Leirvik, 2018).

Con respecto a experiencias formativas en educación secundaria, una investigación española desarrollada en Murcia y Cádiz, demostró la carencia formativa de competencias interculturales de los profesores y reveló la necesidad de implementarlas, tanto en la formación inicial universitaria como en la formación continua (Escarbajal y Morales, 2016). Asimismo, un estudio desarrollado en México, analizó las prácticas de enseñanza de 37 profesores que imparten docencia en centros de secundaria de la comunidad indígena yaqui. Se evidenció un escaso desarrollo de actividades que promueven competencias interculturales y un trabajo cooperativo entre los alumnos (Fernández et al., 2016). Finalmente, un estudio español analizó la adquisición de la competencia intercultural por parte de los profesores de español en formación, a través de metodologías de aprendizaje activo. El análisis realizado demostró que el aprendizaje basado en problemas es el que mejor favorece la adquisición de esta competencia (Hernández, 2016).

En el ámbito universitario, un estudio desarrollado en Reino Unido mostró la importancia concedida por el profesorado a la competencia intercultural como también la falta de familiarización con la misma (Shestakova et al., 2016). Un estudio similar en Chile, demostró que son escasas las ocasiones en donde se aclara cuáles son los contenidos por enseñar y las competencias que debe desarrollar un profesional de la educación al intervenir en escenarios interculturales (Morales-Saavedra et al., 2018). Lo anterior, también fue resaltado por otra investigación en Reino Unido, en la cual se examinó el poder de las relaciones sociales en la implementación de la competencia comunicativa intercultural en un entorno donde se habla inglés como lengua extranjera. A partir de este estudio, se pudo observar cómo la educación intercultural todavía se percibe de manera diferente 
(Loo et al., 2019). En Finlandia, se es un desafío significativo para la examinó el papel del aprendizaje educación en historia, a menudo ubicada experiencial en el desarrollo de en discursos construidos a lo largo del competencias interculturales en el contexto tiempo que trastocan la forma en que las de la formación del profesorado. Para ello, se pidió a los estudiantes locales y extranjeros que estudiaban para convertirse en maestros, que escribieran cinco narraciones breves, cada una de ellas, sobre encuentros interculturales significativos que experimentaron antes de inscribirse en un curso intercultural. El análisis del discurso de las narrativas de los estudiantes mostró que en la mayoría de los casos ya se había producido un aprendizaje intercultural importante antes de que estos estudiantes se embarcaran en el curso (Dervin, 2017). Un estudio en Kayseri (Turquía) buscó determinar los factores que fomentan la competencia intercultural de los futuros profesores de Geografía. Para lo anterior, se realizó una excursión de campo multicultural durante una semana con ocho estudiantes alemanes y ocho turcos. Los resultados indican claramente que el trabajo de campo multicultural fomenta el aprendizaje intercultural a nivel personal (Brendel et al., 2016). En este sentido, un estudio desarrollado en Reino Unido revela el efecto transformador de los encuentros interculturales de los futuros profesores con estudiantes extranjeros (Senyshyn, 2018).

cosas siempre se han enseñado (Garrard, 20201. Por su parte, un artículo sobre los profesores del Estado Brasileño de Roraima puso de relieve el debate sobre la educación continua de los profesores desde la perspectiva de la interculturalidad, que es esencial para que identifiquen, reconozcan y trabajen con la diversidad presente en sus aulas (Nunes y Lima, 2020).

De la misma forma, un estudio asiático aplicó una encuesta a 75 profesores de inglés que enseñan a nivel universitario en cinco universidades del sector público y privado en Pakistán. Los resultados señalan que estos fomentan la incorporación del conocimiento de la interculturalidad en las clases. Sin embargo, existe la necesidad de organizar sesiones de capacitación, pues muchos maestros informaron que no utilizan tales habilidades en sus aulas. Este estudio destaca además que los docentes también podrían beneficiarse de trabajar juntos para desarrollar la enseñanza de la interculturalidad y la competencia transcultural y hacer que este desarrollo suceda en relación con el desarrollo profesional docente (Aziz et al., 2020). Otro estudio, también referido a la Por otra parte, una investigación sobre el enseñanza del Inglés pero en Reino Unido, estado del portugués intercultural en la enfatiza la importancia de que la carrera de Educación Intercultural de la formación del profesorado responda a las Universidad Federal de Goiás muestra que nuevas demandas de este mundo el diálogo intercultural descolonial en las globalizado, proponiendo, entre varios relaciones de poder lingüístico no aspectos, nuevas posturas políticas y prescinde de las estrategias de pedagógicas que conduzcan a preparar a dominación de las propias fuerzas los estudiantes para que sean más críticos dominantes (Rezende y Rodrigues, 2020). con sus propias realidades y más sensibles Asimismo, un estudio realizado en a la interculturalidad (Siqueira, 2017). Australia sostiene que la interculturalidad Finalmente, un trabajo brasileño evidenció 
la necesidad de reflexionar respecto de la innovación pedagógica en Instituciones de la Enseñanza Superior (IES), sobre todo en la formación de profesores que enseñarán para un contexto educativo caracterizado por el respeto a la diversidad, la inclusión e interculturalidad (Mendes y Lustosa, 20181.

Resulta evidente la importancia que las investigaciones atribuyen al desarrollo de la competencia intercultural en la formación del profesorado y en la enseñanza que se lleva a cabo en los diferentes niveles, desde la educación primaria a la terciaria. En general es posible apreciar que falta mucho por avanzar en la incorporación de esta competencia en los procesos de formación inicial docente y en el posterior ejercicio profesional en las salas de clases. Sin perjuicio de lo anterior, es posible reconocer una disposición positiva de los actuales y futuros profesores frente a la diversidad, al contacto con otros y a desarrollar prácticas interculturales en sus aulas, lo cual, sin lugar a dudas, favorecerá el protagonismo de esta competencia.

\section{CATEGORÍA: EDUCACIÓN INTERCULTURAL}

Un estudio desarrollado en Polonia presenta una evaluación de los logros y problemas de la pedagogía intercultural como una (sub) disciplina educativa importante. Concluye, después de un vasto análisis, que la comprensión y aceptación de la educación intercultural depende de muchos factores, como por ejemplo, las experiencias históricas, el nivel de desarrollo de las sociedades y el sistema sociopolítico (Ogrodzka-Mazur, 2018).
Asimismo, se encontró un estudio colombiano donde su propósito fue presentar un panorama teórico con respecto a la relación biopedagógica y educación intercultural. Los resultados arrojados por la investigación permitieron caracterizar cómo los procesos cognitivos son procesos biológicamente auto organizados, de alta complejidad, y de creación permanente y que se enriquecen en entornos interculturales (Medina, 2017). Otro trabajo, también de aquel país, presenta una reflexión, desde la Filosofía de la Educación, sobre la situación de la educación intercultural en la sociedad global. Se expone la situación actual de dicha forma de educación, y se enuncian y examinan los principios (diálogo crítico y sociedad de la cooperación), finalidades /construcción de identidad, construcción de racionalidad, construcción de personalidad), y acciones (prácticas sociales) esenciales para la formación del profesorado en esta modalidad educativa, así como la necesidad de incluir en ella la formación investigativa (Lafuente, 2017). Asimismo, un estudio argentino cuestiona el modelo de pensamiento que subyace a la colonialidad del conocimiento en la formación docente en contextos latinoamericanos. A partir del replanteamiento de las bases epistemológicas y metodológicas en los procesos formativos educativos, reconoce que es a partir del encuentro y diálogo entre diferentes racionalidades que la formación del profesorado en la perspectiva de la interculturalidad se abre a una posición crítica, ética y política en defensa de la dignidad y libertad humana y sus diversas formas de ser y hacer (Cecchetti et al., 2020).

Además, una investigación desarrollada en Chile estableció que la formación inicial docente en educación intercultural no sólo se circunscribe a la educación primaria y 
secundaria sino que también a la pre-escolar. En este sentido, el estudio develó que en las familias y comunidades indígenas mapuches presentes en Chile existen conocimientos asociados a prácticas socioculturales, que permitirían sustentar una Educación Parvularia, desde un enfoque educativo intercultural, con base epistémica en la memoria social de padres y sabios. Lo anterior implica que se deben generar instancias académicas formales e informales que apunten a que el estudiante aprenda a entablar una relación no conflictiva con personas de otras culturas. Morales-Saavedra et al., 20181.

Por otra parte, las investigaciones revisadas destacan la importancia del currículo para el desarrollo de una educación intercultural. Un estudio español sostiene que éste puede aportar en la formación para la ciudadanía intercultural a la vez que puede reorientar las prácticas pedagógicas. En este sentido, para elaborar un currículum inclusivo e intercultural es necesario recoger todas las reflexiones teóricas de diferentes disciplinas y establecer puentes entre las diferentes materias y reformular los contenidos, las metodologías, la organización de tiempos y espacios, como también la evaluación (Louzao et al., 2020). Lo anterior cobra fuerza al considerar los resultados de una investigación desarrollada en Perú, que analizó las percepciones de los egresados de la Facultad de Educación en la Universidad Nacional Mayor de San Marcos respecto de su formación pedagógica en el marco de la educación intercultural. Entre los principales resultados, se encuentra que tanto la programación curricular como las metodologías empleadas por los docentes no consideran ni incluyen los principios de a pedagogía intercultural. Entre las conclusiones obtenidas, destaca que rige un currículum de corte monocultural y reproductor del modelo hegemónico, pese a que la característica principal de dicha población estudiantil es su diversidad cultural y lingüística (Sotomayor, 2020).

Una encuesta realizada a 189 estudiantes de la Escuela Normal Superior de México, buscó explorar la importancia percibida del contacto con "lo extranjero" como parte de la formación magisterial, el interés existente por la participación en algún programa de intercambio internacional y el nivel de experiencias concretas con "lo extranjero". A partir de lo que demuestran las percepciones, intereses y experiencias de los futuros maestros de secundaria, se manifiesta la importancia de un mayor compromiso institucional y gubernamental con la formación intercultural del magisterio normalista (Pöllmann, 2018).

Con respecto a esta categoría, las investigaciones dan cuenta de una importante revisión histórica, filosófica, conceptual, ontológica y epistemológica sobre lo que vamos a entender como educación intercultural. También es posible apreciar la necesidad de que este tipo de educación se manifieste en todos los procesos formativos y se exprese en todos los niveles escolares, incorporando también a la comunidad de los territorios en donde se encuentran emplazados los establecimientos escolares y de educación superior. En este sentido, se demanda un currículo escolar y universitario que incorporé la multiculturalidad y propicie una interculturalidad que contribuya a desplazar los currículos monoculturales aún predominantes. 


\section{CATEGORÍA: PRÁCTICAS DE ENSEÑANZA}

Un estudio llevado a cabo en España identificó que las y los docentes tienen un entendimiento y una actitud positiva hacia la interculturalidad y tratan de incluirla en su práctica pedagógica en aula. Sin embargo, manifiestan la necesidad de ampliar su conocimiento y contar con herramientas pedagógicas para enseñar la primera y la segunda lengua, cómo hacer dialogar las diferentes culturas en cada área curricular y cómo trabajar la interculturalidad transversalmente (Escarbajal y Morales, 2016).

También es posible encontrar algunas investigaciones latinoamericanas. En Chile, un estudio realizado en escuelas situadas en contextos interculturales de la Araucanía reveló que las prácticas de enseñanza de profesores interculturales y tradicionales se encuentran focalizadas en las clases expositivas, evidenciándose una ausencia de articulaciones pedagógicas y prácticas reflexivas, lo cual dificulta los procesos de enseñanza-aprendizaje (Beltrán-Véliz et al., 2019). En esta misma línea, otra investigación chilena concluye que el trabajo de los Educadores Tradicionales dentro de las escuelas es aislado y carece del apoyo de las comunidades locales y del Programa de Educación Intercultural Bilingüe (Becerra-Lubies et al., 2019). Asimismo, una investigación analizó discursos de personas mapuche y aymara, en relación a la formación docente para así contribuir a una formación para la atención de la diversidad cultural en las carreras de Pedagogía, cuestión que consideramos urgente (lbáñez-Salgado y Druker-lbáñez, 2018). Por otra parte, una indagación argentina en escuelas destinadas a niños pertenecientes a comunidades originarias en el norte de la provincia de Salta, permitió comprender cómo la experiencia educativa intercultural se encarna en los cuerpos de los/as sujetos/as, rastreando las formas en que el orden sociopolítico y educativo produce las subjetividades en sí (Bazan y Tejerina, 2018). Finalmente, un trabajo brasileño relacionado con los Derechos Humanos, Educación, Interculturalidad reflexionó sobre la construcción de prácticas pedagógicas desde una perspectiva intercultural capaz de movilizar prácticas educativas orientadas a una educación crítica (Sacavino, 2020).

También fue posible recopilar investigaciones desarrolladas en los continentes asiático y europeo. En el primer caso, se evidenció que los maestros vietnamitas muestran un alto grado de interacción y disfrute, así como respeto por las diferencias culturales. Sin embargo, sus niveles de confianza en la interacción y atención son moderados. Las conclusiones sugieren que los profesores necesitan desarrollar su competencia intercultural especialmente en términos de habilidades prácticas (Gómez-Parra y Há, 2021). Por su parte, en Europa un primer estudio indagó sobre las formas en que la identidad profesional de los docentes en Chipre está siendo moldeada e influenciada en entornos escolares muy diversos. Se encontró que la falta de capacitación y recursos, la falta de respeto y la negligencia de las experiencias, ideologías, valores y creencias previas que afectan la identidad de los maestros $y$, en consecuencia, la promulgación de políticas interculturales (Karousiou et al., 2018). Por su parte, una investigación desarrollada en los Balcanes occidentales estableció el potencial del liderazgo docente como una estrategia para promover la educación intercultural al empoderar a los padres y miembros de grupos étnicos minoritarios para participar en la vida de la escuela (Vranješević y Frost, 2016). Finalmente, una 
investigación desarrollada en España, analizó las creencias que tienen los estudiantes universitarios de educación sobre la noción de interculturalidad y sobre cómo han de ser abordadas las prácticas educativas en contextos multiculturales. El estudio concluye que la mayoría de los estudiantes identifican la interculturalidad con la inmigración, que a su vez asocian con problemas o déficits, sobre todo lingüísticos y de comunicación (Rodríguez-lzquierdo, 2016 ).

En esta categoría, se evidencia que los profesores tienen una actitud positiva hacia la interculturalidad y hacen esfuerzos por incluirlas en sus prácticas pedagógicas, pero también manifiestan la necesidad de ampliar su conocimiento sobre esta temática y contar con herramientas pedagógicas que permitan movilizarla a la sala de clases. En este sentido, las investigaciones dan cuenta de una ausencia de articulaciones pedagógicas y prácticas reflexivas que dificultan los procesos de enseñanza aprendizaje desde un enfoque intercultural.

\section{CONCLUSIÓN}

La revisión por alcance realizada permite evidenciar que existe una importante ausencia de investigaciones en torno a educación geográfica intercultural tanto a nivel nacional como internacional. A diferencia de lo señalado, si fue posible encontrar un importante número de estudios referidos a interculturalidad y educación intercultural, en diversos países y continentes, como también, pero en menor cantidad, sobre didáctica y enseñanza de la geografía. Lo anterior, abre un importante campo de investigación en torno a esa temática, que contribuiría a la formación inicial docente y a la formación continua de los profesores en ejercicio, sobre todo para quienes desarrollan su labor pedagógica en espacios geográficos multiculturales.

trabajo da respuesta a las interrogantes formuladas al comienzo de éste. Los temas más publicados para la dimensión educación geográfica se refieren a temáticas pedagógicas, disciplinarias y didácticas. En cuanto a la dimensión intercultural, éstas se refieren a la competencia intercultural, la educación geográfica y prácticas de enseñanza interculturales. Los países que concentran las mayores publicaciones son Reino Unido y España, en Europa; Chile, Brasil y México, en América.

Lo anterior cobra mayor importancia aún si consideramos cómo las aulas escolares son cada vez más diversas y multiculturales, donde conviven estudiantes de múltiples nacionalidades y realidades culturales. Esto genera escenarios educativos complejos que requieren docentes preparados para desarrollar clases en un ambiente intercultural $y$, por ende, inclusivo que recojan e integren los saberes de todos sus estudiantes sin distinción alguna. De este modo, los resultados obtenidos en este estudio también nos llevan a repensar la formación inicial docente $y$, por tanto, el diseño curricular, pedagógico y didáctico de las Instituciones de Educación Superior que forman a los futuros profesores. Lo anterior, porque las investigaciones demuestran que queda aún mucho camino por recorrer para dotar de competencias interculturales a quienes se forman como maestros.

En el actual contexto educativo, la educación geográfica tiene mucho que aportar en la formación del futuro ciudadano, que valore las diferencias culturales entre quienes habitan un territorio y así generar dinámicas espaciales de convivencia intercultural sustentadas en el 
respeto y valoración por el otro. En este sentido, la construcción de una educación geográfica intercultural pueda aportar no sólo a la comprensión de las dinámicas socio espaciales de un determinado espacio sino que también puede contribuir a construir puentes para una convivencia tolerante y pacífica en el espacio geográfico. Lo anterior, cobra mayor fuerza en territorios como la región de la Araucanía, caracterizada estos últimos años por conflictos territoriales y sociales entre sujetos de diferentes culturas. Además, es posible constatar la permanen cia de una escuela y un currículum monocultural que desconoce e invisibiliza los conocimientos ancestrales y actuales de los descendientes de la población originaria mapuche. A todo lo anterior se suma, la incorporación en las escuelas de la región de niños y jóvenes provenientes de otros países latinoamericanos, producto de los procesos de inmigración actuales, lo que nos permite observar en las salas de clases a estudiantes provenientes de Haití, Venezuela y Colombia. Esta compleja realidad abre múltiples posibilidades pedagógicas y didácticas para educar en la diversidad y a partir del conflicto.

Por lo anterior, se le presenta a la Didáctica de la Geografía una oportunidad para indagar sobre estas realidades multiculturales de modo de generar conocimiento didáctico que permita conocer como recoger e integrar los conocimientos, habilidades y actitudes de estudiantes provenientes de múltiples realidades territoriales y culturales y cómo plasmarlas en procesos de enseñanza aprendizaje que contribuyan no sólo al desarrollo de una disciplina en particular sino también a una convivencia intercultural en diferentes espacios geográficos.

Por otra parte, la revisión de literatura por alcance se transforma en una importante herramienta para iniciar el estudio de una determinada temática y conocer el nivel de producción que existe en torno a ésta, como se ha reportado en este estudio. Asimismo, las revisiones por alcance también pueden ser útiles precursores de revisiones sistemáticas y pueden usarse para confirmar la relevancia de los criterios de inclusión y las posibles preguntas. En esta ocasión, los resultados obtenidos nos abren una invitación a realizar una revisión sistemática sobre educación geográfica intercultural que nos permita avanzar en el propósito de la revisión, en identificar las lagunas de conocimiento, analizar un cuerpo de literatura y aclarar conceptos, entre otros. Lo anterior, nos llevaría a superar las limitaciones de esta investigación que sólo consideró artículos relacionados con las temáticas involucradas, descartando otras formas de difusión como los libros, capítulos de libros, tesis, actas y ponencias de congresos.

Finalmente, este estudio deja en evidencia la necesidad de avanzar, por parte de la Didáctica de la Geografía, en estudios sobre cómo enseñar geografía en contextos multiculturales e interculturales de modo de poder ir desarrollando y propiciando enfoques didácticos de enseñanza geográfica intercultural inclusivos. Lo anterior vendría a responder una demanda hoy latente que es contar con una educación geográfica que contribuya a formar ciudadanos con competencias geográficas e interculturales, para así generar espacios geográficos sostenibles e inclusivos. Por lo anterior, la pregunta de investigación para futuras investigaciones es ¿Cómo desarrollar una educación geográfica intercultural en Chile? 


\section{REFERENCIAS BIBLIOGRÁFICAS}

Alomar-Garau, G., Gómez-Zotano, J., Arias-García, J., y Arias, J. (2017). Teaching landscape in Spanish universities: looking for new approaches in Geography. Journal of Geography in Higher Education, $41(2), \quad$ 264-282. hitps:// doi.org/10.1080/03098265.2017.12 95226

Applis, S. (2016). Geography teachers concepts of working with Thinking Through Geography strategies-results of an empirical reconstructive study. International Research in Geographical and Environmental Education, 25(3), 195-210. hitps://. doi.org/10.1080/10382046.2016.11 55326

Araya, F., y De Souza, L. (2018). Desarrollo del pensamiento geográfico: un desafío para la formación docente en Geografía. Revista de Geografía Norte Grande, 70, 51-69. https://scielo.conicyt.cl/pdf/rgeong/n70/0718-3402-rgeong-70-0005 1.pdf

Araya, F. (2010). Educación geográfica para la sustentabilidad. Editorial Universidad de La Serena.

Araya, F., y Álvarez, S. (2019). Desarrollo del pensamiento geográfico y formación ciudadana: Una mirada desde el espacio vivido. Signos Geográficos, 1, 3-18. https://www.revistas. ufg.br/signos/article/download/59376/33345

Arenas, A., y Salinas, V. (2013). Giros en la Educación Geográfica: renovación de lo geográfico y lo educativo. Revista de Geografía Norte Grande, 56, 143-162. https://scielo.conicyt.cl/pdf/rgeong/n56/art08.pdf
Arenas, A., Figueroa, R., y Salinas, V. (2018). Educación geográfica y formación ciudadana. BAURU, 6(2), 17-66. https://www. researchgate.net/publication /332089100_Educacion_geografica_y_f ormacion_ciudadana

Aziz, F., Saleem, T., Saleem, A., y Batool, R. (2020). From pragmatic competence to intercultural competence: Pakistani ESL teachers perspectives of teaching interculturality in ESL classroom. Revista Amazonia Investiga, 9(33), 37-45. https://doi.org/10.34069/ai/2020.33.09.4

Bazan, M. D., y Tejerina, M. (2018). Educación intercultural bilingüe: narrativizando subjetividades docentes. Revista Del CISEN Tramas/Maepova, 6(2), 173-191. https://dialnet.unirioja.es/servlet/articulo? codigo $=6945566$

Becerra-Lubies, R., Bolomey, C., y Meli, D. (2019). Teaching indigenous languages in Chile: perceptions of Traditional Educators. International Journal of Bilingual Education and Bilingualism, 1-19. https://doi.org/10.1080/13670050.2019.16 21260

Becerra, R. y Llanquinao, G. (2017). Mapu Kimün. Relaciones mapunche entre persona, tiempo y espacio. Ocho Libros Editores.

Beltrán- Véliz, J. C., Mansilla- Sepúlveda, J. Valle- Rojas, C., y Navarro- Aburto, B. (2019). Prácticas de enseñanza de profesores en contextos interculturales: obstáculos y desafíos. Magis, Revista Internacional de Investigación En Educación, 17(23), 11-23. https://doi.org/10.1 1 144/Javeriana.m 1 1-23.pepc

Béneker, T., y Palings, H. (2017). Student teachers' ideas on (powerful) knowledge in 
geography education. Geography, 102(2), 79-85.

Brendel, N., Aksit, F., Aksit, S., y Schrüfer, G. (2016). Multicultural group work on field excursions to promote student teachers' intercultural competence. Journal of Geography in Higher Education, 4021, 284-301. hittps://doi.org/10.1080/03098265.2016.1140 731

Brooks, C. (2017). Pedagogy and identity in initial teacher education: developing a "professional compass." Geography, 102(1), 44-50.

Brysch, C. P. (2020). Teacher Attitudes toward Alternative Professional Development in Geography. Journal of Geography, 119(2), 55-62. https://doi.org/10.1080/00221341.2019.17 06621

Cecchetti, E., Pozzer, A., y Tedesco-Anderson, L. (2020). Formação Docente Intercultural e Colonialidade do Saber. Revista Del CISEN Tramas/Maepova, 8(1), 187-200. Disponible: https://ppct.caicyt.gov.ar/index.php/cisen/article/view/16487

Dervin, F. (2017). "I find it odd that people have to highlight other people's differences - even when there are none": Experiential learning and interculturality in teacher education. International Review of Education, 63(1), 87-102.

Escarbajal, A., y Morales, A. (2016). Estudio de las competencias interculturales del profesorado en Educación Secundaria. IJERI: International Journal of Educational Research and Innovation, 6, 146-161. https://dialnet.unirioja.es/servlet/articulo? codigo $=5732761$ \&info=resumen\&idioma $=$ SPA
Fernández, M., Arenas, E., y Hernández, C. (2016). Creencias sobre la educación intercultural y prácticas de enseñanza de profesores de secundaria de la población indígena yaqui. Perfiles Educativos, 38(152), 109-127. http://www.scielo.org.mx/scielo.php? script=sci_arttext\&pid=S0 1 85-269820160002001 09\&la $\mathrm{ng}=\mathrm{es}$

Fögele, J., Luber, L., y Mehren, R. (2019). Types of student teachers between theory and practice in the subject of geography: reconstructing orientations using the example of a seminar on experiments. Journal of Geography in Higher Education, 44(2), $\begin{array}{llllll}3 & 1 & 0 & - & 3 & 3\end{array}$ https://doi.org/10.1080/03098265.20 19. 1698524

García, F. (2018). Introducción a las revisiones sistemáticas de literatura. España: Universidad de Salamanca. Grupo de Investigación en InterAcción y elearning (GRIAL), 1-4. htips://doi.org/10.5281/zenodo. 1193285

Garrard, K. (2020). Finding a way in for interculturality: analysing History teachers' conceptualisations at the secondary school level. Discourse: Studies in the Cultural Politics of Education, 1-13. https://doi.org/10.1080/01596306.2020.18 25288

Garrido, M. (2005). El espacio por aprender, el mismo que enseñar: las urgencias de la educación geográfica. Cad. Cedes, Campinas, 25(66), 137-163. https://www.scielo.br/i/ccedes/a/KBTgrHS-

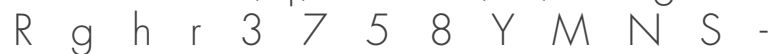
$v S 6 D /$ ? lang=es\&format=pdf

Garrote, D., Arenas, J. Á., y Jiménez-Fernández, S. (2018). Educación intercultural en el aula: Profesora y TIC. Revista Prisma Social, 22, 326-345. 
https://revistaprismasocial.es/article/view $/ 2580$

Golightly, A. (2021). Self-and peer assessment of preservice geography teachers' contribution in problem-based learning activities in geography education Self-and peer assessment of preservice geography teachers' contribution in problem-based learning activities in geography e. International Research in Geographical and Environmental Education, 3011), 75-90. https://doi.org/10.1080/10382046.2 020.1744242

Gómez- Parra, M. E., y Há, T.-A. (2021). Primeros pasos hacia una educación intercultural en Vietnam: explorando la competencia intercultural de los maestros de primaria. Culture and Education, 33(1), $\begin{array}{llllll}7 & 8 & - & 1 & 0 & 5\end{array}$. https://doi.org/10.1080/11356405.2 020.1859740

Harte, W. (2017). Preparing Preservice Teachers to Incorporate Geospatial Technologies in Geography Teaching. Journal of Geography, $116151,226-236$. https://doi.org/10.1080/00221341.2 017.1310274

Hernández, N. (2016). Adquisición de la competencia intercultural y metodologías de aprendizaje activo: un estudio sobre la formación de profesores de español. Journal of Spanish Language Teaching, 3(1), 1-14. https://doi.org/10.1080/23247797.2016.11630 39

Ibáñez-Salgado, N., y Druker-lbáñez, S. (2018). Intercultural education in Chile from the perspective of the actors: A co-construction. Convergencia, 25(78), 227-249. $\mathrm{h}+\mathrm{t} \mathrm{p} \mathrm{s}$ : / / doi.org/10.29101/crcs.v25i78.9788
Karolčík, Š., Čipková, E., y Mázorová, H. (2016). Application of digital technologies in the geography teaching process from the teachers' perspective. International Research in Geographical and Environmental Education, 25(4), 328-343. https://doi.org/10.1080/10382046.2016.12 07992

Karousiou, C., Hajisoteriou, C., y Angelides, P. (2018). Teachers' professional identity in super-diverse school settings: teachers as agents of intercultural education. Teachers and Teaching Theory and Practice, 25(2), 240-258. https://doi.org/10.1080/13540602.2018.15 44121

Lafuente, M. I. (2017). Educación intercultural y formación del profesorado. Revista Historia de La Educación Latinoamericana, 19(29), 89-109. https://doi.org/10.19053/01227238.7554

Loo, D., Trakulkasemsuk, W., y Pattamawan, J. (2019). Examining power through social relations in teachers' discourse about intercultural education. Intercultural Education, 30(4), 398-414. https://doi.org/10.1080/14675986.2018.15 40110

López, L. E. (2001). La cuestión de la interculturalidad y la educación latinoamericana. Documento de trabajo presentado en el Seminario sobre prospectivas de la educación en la región de América Latina y el Caribe. UNESCO-Santiago. https://red.pucp.edu.pe/ridei/wp-content/uploads/biblioteca/inter44.PDF

Louzao, M., Francos, M. C., y Verdeja, M. (2020). Educación intercultural: algunas reflexiones y orientaciones para una práctica pedagógica en una escuela democrática y plural. Estudios Pedagógico, 46(1), 
$431-450$

https://- Morales-Saavedra, S., Quintriqueo, S. doi.org/10.4067/S0718-0705202000 Uribe-Sepúlveda, P., y Arias-Ortega, K. 0100431

(2018). Interculturalism in higher education: experience in preschool education in La

Lozano, P. (2010). Las salidas a terreno Araucanía, Chile. Revista de Ciencias como posibilidad de enseñanza de la Sociales, (77), 55-76. https://geografía: Relatos de experiencia en la doi.org/10.29101/crcs.v25i77.4706 ciudad de Santiago, Chile. Geosaberes, 7(2), 72-90. https://www.reda- Moreno, R., y Atienza, E. (2016). Abordar lyc.org/pdf/5528/552856443006.pdf la interculturalidad en el aula desde el

Llancavil, D. (2018). Trabajo de Campo: desarrollo de la competencia intercultural Producción y Significancia de Conocimiento Geográfico. Perspectiva Educacional. Formación de Profesores, 57/21, 143-162. http://www. perspectivaeducacional.cl/index.php/peducacional/article/view/625 del profesorado. Revista de Didáctica Español Como Lengua Extranjera, (22), 1-24. https://marcoele.com/descargas/22/moreno-atienza_interculturalidad.pdf

Müun, Z., Peters, M., Stern, C., Tufanaru, Martínez, L. (2017). La enseñanza de la geografía y la formación geográfica en los estudios universitarios de maestro. Tabanque. Revista Pedagógica, 30, 195-217. https: //dialnet.unirioja.es/servlet/articulo? codigo=6 138673

Medina, R. (2017). Bioaprendizaje y educación intercultural. Sophia, 1311), 47-54. https://doi.org/10.18634/sophiaj. $13 v .1 \mathrm{i} .686$

C., McArthur, A. y Aromataris, E. (2018). Systematic review or scoping review? Guidance for authors when choosing between a systematic or scoping review approach. BMC Medical Research Methodology. 181143), 1-7. https://researchers.mq.edu.au/en/public ations/systematic-review-or-scoping-reviewguidance-for-authors-when-cho

Novaro, G. (2006). Educación intercultural en la Argentina: potencialidades y riesgos. Mendes, J., y Lustosa, F. (2018). Inno- Ponencia presentada en Foro de Educación vación Pedagógica, Inclusión e Interculturali- Mundial, Buenos Aires.

dad en la Formación de Profesores en Pedagogía y Magisterio Indígena en la Universi- Nguyen, T. P. (2018). Education for Sustaindad Federal de Ceará. Revista Ibero-Ameri- able Development in Vietnam: exploring the cana de Estudos Em Educação, 13(2), geography teachers' perspectives. Interna1281-1300. https://- tional Research in Geographical and Environdoi.org/10.21723/riaee.v13.ne-mental Education, 27(4), 341-356. sp2.set2018.11644

Miranda, P. (2012). La educación geográfih $\mathrm{t}+\mathrm{p}$ s : / / doi.org/10.1080/10382046.2017.136 ca en Chile: desde su aparición en el currículum escolar en el siglo xix hasta los ajustes Nunes, C., y Lima, M. (2020). Formação curriculares de 2010. Anekumene, (4), continuada de professores e interculturali51-71. https://doi.org/10.17227/aneku- dade: reflexões sobre aspectos multiétnicos mene.2012.num4.7538 do ensino em língua portuguesa. E-Curricu 
lum, 18(3), 1178-1196. https://doi.org/10.23925/1809-3876.2020v 1 8i3p $1178-1196$

Ogrodzka-Mazur, E. (2018). Intercultural Education in Poland: Experiences, Problems and Prospects. En Bernard, C. y Llevot-Calvet, N. (Eds.) New Pedagogical Challenges in the 21 st Century. Contributions of Research in Education (pp.7-71). https://doi.org/10.5772/intechopen.71010

Pöllmann, A. (2018). La formación intercultural de los futuros maestros Mexicanos de secundaria. Revista Electrónica de Investigación Educativa, 2011), 83-92. https://doi.org/10.24320/redie.2018.20.1.14 61

Puttick, S., y Warren-Lee, N. (2021). Geography mentors' written lesson observation feedback during initial teacher education. International Research in Geographical and Environmental Education, 30(2), 95-111. $\mathrm{h}+\mathrm{t} \mathrm{p}$ : / / doi.org/10.1080/10382046.2020.17 57830

Quintriqueo, S., Quilaqueo, D., Gutiérrez, M. y Peña, F. (2015). Enseñanza de Historia, Geografía y Ciencias Sociales: hacia una Perspectiva Intercultural. Ediciones Universidad Católica de Temuco.

Quintriqueo, S. y Arias, K. (2019). Educación intercultural articulada a la episteme indígena en Latinoamérica. El caso mapuche en Chile. Diálogo Andino, (59), 81-91. http://dialogoandino.cl/wp-content/uploads/2019/08/06-QUINTRIQUEO-RDA59.pdf

Rezende, T., y Rodrigues, E. da R. (2020). Perspectiva intercultural em práticas de formação de docentes indígenas. Revista Linguagem \& Ensino, 23(4), 1204-1223. https://doi.org/10.15210/rle.v23i4. 18551

Rodríguez-lzquierdo, R. (2016). Creencias acerca de la interculturalidad y las prácticas educativas interculturales de los estudiantes universitarios de educación social en el contexto español. Perfiles Educativos, 38(152), 128-154. hitp://www.scielo.org. mx/scielo.php?script=sci_arttext\&pid=S0 1 85-269820 $16000200128 \& 1 a$ $\mathrm{ng}=\mathrm{es}$

Sacavino, S. (2020). Interculturalidade e Práticas Pedagógicas: construindo caminhos. Educação (UFSM), 45, 1-18. https://doi.org/10.5902/1984644438 257

Salinas, V., y Brooks, C. (2018). Expandiendo la noción de profesionalidad docente desde la educación geográfica. Revista de Geografía Norte Grande, 70, 85-108. https: / / scielo.conicyt.cl/pdf/rgeong/n70/071 8-3402-rgeong-70-0008 5.pdf

Scholten, N., Höttecke, D., y Sprenger, S. (2019). How do geography teachers notice critical incidents during instruction? International Research in Geographical and Environmental Education, 29(2), 163-177. https://doi.org/10.1080/10382046.2 019.1670915

Senyshyn, R. (2018). Teaching for transformation: converting the intercultural experience of preservice teachers into intercultural learning. Intercultural Education, 29(2), 163-184. https://doi.org/10.1080/14675986.2018.14 29791

Shestakova, N., Domínguez, M., y Cacheiro, M. L. (2016). Competencias del profesorado universitario para la intercultura 
lidad. Acción Pedagógica, 25, 74-87. https: / / dialnet.unirioja.es/descarga/articulo/6224929.pdf

Siqueira, S. (2017). Intercultural language educators for an intercultural world: action upon reflection. Intercultural Education, 28(4), 390-407. https://doi.org/10.1080/14675986.2017.13 34396

Sotomayor, Y. (2020). Percepción de los egresados sanmarquinos respecto de su formación pedagógica en el marco de la educación intercultural. PLURIVERSIDAD, 6, $\begin{array}{llllll}1 & 3 & - & 1 & 6 & 2\end{array}$. https://doi.org/10.31381/pluriversidad .vOi6.3637

Svobodová, H., Mísařová, D., Durna, R., y Hofmann, E. (2020). Geography Outdoor Education from the Perspective of Czech Teachers, Pupils and Parents. Journal of Geography, 119(1), 32-41. $\mathrm{h}+\mathrm{t} \mathrm{p} \mathrm{s}: \mathrm{l} /$ doi.org/10.1080/00221341.2019.1 694055
Turra, O. (2017). Historia escolar en contexto interétnico e intercultural Mapuche. Educ. Soc., Campinas, 38(140), 791-808. https: / / www.redalyc.org/pdf/873/87353321015.pdf

Vea, E., y Leirvik, B. (2018). Interculturally competent teachers in the diverse Nonwegian educational setting. Multicultural Education Review, 10(4), 274-291. https://doi.org/10.1080/2005615X.2018.1532 223

Vraniešević, J., y Frost, D. (2016). Stories From Intercultural Education in Serbia: Teacher Leadership and Parent Participation. European Education, 48(1), 63-78. hitps://doi.org/10.1080/10564934.2016.11539 32

Williamson, G. y Pérez, I. (2017). Oficios de la tierra: Diálogo de saberes en la educación ambiental intercultural. En E.Triviño, L.Morawietz, C.Villalobos y E.Villalobos (Eds.) Educación Intercultural en Chile. Experiencias, pueblos y territorios (pp.333-366). Ediciones Universidad Católica. 